\title{
Caspase-3, p53 and Bcl-2 expression in basal cell carcinoma of the eyelid
}

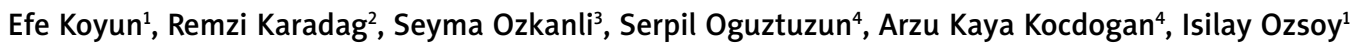

'Department of Ophthalmology, Kastamonu State Hospital, Kastamonu, Turkey

2Department of Ophthalmology, School of Medicine, Istanbul Medeniyet University, Goztepe, Istanbul, Turkey ${ }^{3}$ Department of Pathology, School of Medicine, Istanbul Medeniyet University, Goztepe, Istanbul, Turkey

${ }^{4}$ Department of Biology, School of Medicine, Kirikkale University, Kirikkale, Turkey

Adv Dermatol Allergol 2020; XXXVII (4): 535-539

DOI: https://doi.org/10.5114/ada.2020.98285

\begin{abstract}
Introduction: Eyelid tumours mostly originated from skin and its appendeges. External carcinogens like UV radiation causes cell damages in the eyelid skin and contributes to carcinogenesis. Apoptosis is a very important mechanism to prevent these damage and probable neoplatic change.

Aim: To compare caspase-3, p53 and Bcl-2 levels between patients with basal cell carcinoma (BCC) of the eyelid and healthy individuals.

Material and methods: Pathology archives from October 2012 to April 2015 were scanned for BCC biopsies of the eyelid and tissue removed during blepharoplasty and entropion procedures. A total of 36 specimens were found. The specimens were divided into two groups: BCC group and controls (consisting of eyelid tissue removed during routine blepharoplasty). The pathology specimens were then stained using p53, Bcl-2 and caspase-3 stains and the intensity of staining was graded on a 0-3 scale.

Results: Samples from a total of 36 patients were included in the study. Eighteen (50.0\%) patients were female. There were 13 patients in the BCC group and 23 patients in the control group. The mean age was $66.0 \pm 10.8$ years in the BCC group, and $65.61 \pm 11.22$ years in the control group. The caspase- 3 staining was lower in the BCC group than in the control group. No significant differences were found between the BCC group and the control group in terms of p53 levels or Bcl-2 levels (both of them, $p=1.000$ ).

Conclusions: The caspase-3 level was lower in the BCC group. This result suggests that these enzymes can play a significant role in carcinogenesis of eyelid BCC.
\end{abstract}

Key words: basal cell carcinoma, Bcl-2, caspase-3, eyelid tumour, immunohistochemical stain, p53, malignant tumour.

\section{Introduction}

Eyelid tumours originate for the most part in the skin and skin appendages and up to $10 \%$ of these tumours are malignant in nature. Early diagnosis and treatment are paramount in these cases to prevent serious morbidity or death. The most common eyelid malignant tumour is basal cell carcinoma (BCC) [1]. As with skin cancers involving other parts of the body, ultraviolet (UV) radiation is the most significant external risk factor in eyelid cancers [1]. Environmental factors increase oxidative stress, resulting in DNA damage and cell injury. Some of the damage can be repaired by cellular mechanisms so that cells can function normally, but if the damage exceeds cell repair capacity, cells undergo apoptosis or malignant changes [1].

Apoptosis is a necessary process of programmed cell death, playing an important role in both normal and neoplastic tissues. In normal tissues apoptosis leads injured or mutated cells to death and prevent further mutations and carcinogenesis. Changes in the nature of apoptosis may cause a variety of pathological conditions, especially cancers [2].

P53 is a major tumour suppressor protein for apoptosis and cell cycle arrest in DNA damage and cell injury [3]. It presents in the cytosol in low levels when there is no cellular injury and is controlled primarily by murine double minute oncogene (MDM2) [4]. When UV radiation

Address for correspondence: Efe Koyun MD, Department of Ophthalmology, Kastamonu State Hospital, Kastamonu, Turkey, phone: +90 505 8943261, e-mail: efe_koyun@icloud.com Received: 20.12.2018, accepted: 21.01.2019. 
or other environmental factors cause DNA damage, p53 starts to accumulate in the nucleus. It leads to cell cycle arrest and to apoptosis and therefore prevent further mutations and carcinogenesis [5].

Imbalance between cell survival and apoptosis is a key factor in eyelid malignancies and so p53 is an important factor for skin cancers like in other human cancers [6].

$\mathrm{Bcl}-2$ is a protein family that can either promote or inhibit apoptosis [6]. Antiapoptotic subfamily includes Bcl-2, $\mathrm{BCl}-\mathrm{x}, \mathrm{BCl}-\mathrm{w}, \mathrm{Mcl}-1$, and $\mathrm{A} 1$. Bcl-2 supports cell viability by binding pro-apoptotic proteins and suppressing apoptosis. Bcl proteins and carcinogenesis is first studied in $\beta$-cell lymphomas and overexpression of $\mathrm{BCl}-2$ is related to the neoplastic change and expansion $[7,8]$. $\mathrm{Bcl}-2$ protein levels and its alterations are a very important mechanism for carcinogenesis and have been studied extensively over the years, and high levels of $\mathrm{Bcl}-2$ expression show poor prognosis for most of the cancers.

Caspases are a proapoptotic protease family that cleaves important targets for execution of apoptosis [9]. Caspase- 3 is a very important member of this family and induces nuclear changes for apoptosis [10]. Caspase-3 levels are lowered in many types of cancer like cervical and breast cancer and also elevated caspase-3 levels are a positive prognostic factor for gastric cancers [11, 12] Caspase-3 alterations are also related to neurodegenerative diseases like Alzheimer's disease [13] and are also associated with normal neuronal development in animal models [14].

\section{Aim}

The present study is designed to assess p53, Bcl-2 and caspase- 3 levels in BCC of the eyelid and compare them to the p53, Bcl-2 and caspase-3 levels in normal eyelid tissue.

\section{Material and methods}

This retrospective, multicentre study was approved by the Istanbul Medeniyet University ethics committee and adhered to the tenets set forth in the Declaration of Helsinki. Informed consent was obtained from all of the participants before surgery. Ophthalmology and pathology archives were searched at the Istanbul Medeniyet University Goztepe Research and Training Hospital for patients who had undergone eyelid biopsy for eyelid lesions as well as those who had undergone oculoplastic surgeries for entropion, blepharoplasty, or dermatochalasis from 1 October 2012 to 30 April 2015. The samples were divided into 2 groups as malignant and control. Samples from a total of 36 patients were included in the study. Of these, 13 patients had been diagnosed with BCC of the eyelid, and in 23 patients, normal eyelid skin was removed during blepharoplasty procedures.

\section{Immunohistochemical staining}

Immediately after the removal of biopsies, tissues were fixed in $10 \%$ buffered formalin and embedded in paraffin blocks. Sections that were $4 \mu \mathrm{m}$ thick were cut, and one section was stained with haematoxylin-eosin to observe the tissue morphology. For immunohistochemistry, endogenous peroxidase activity was blocked by incubating the sections in $1 \%$ hydrogen peroxide ( $/ \mathrm{v}$ ) in methanol for $10 \mathrm{~min}$ at room temperature (RT). The sections were subsequently washed in distilled water for $5 \mathrm{~min}$, and antigen retrieval was performed for $3 \mathrm{~min}$ using $0.01 \mathrm{M}$ citrate buffer ( $\mathrm{pH}$ 6.0) in a domestic pressure cooker. After washing in distilled water, the sections were transferred in $0.05 \mathrm{M}$ Tris- $\mathrm{HCl}(\mathrm{pH} 7.6)$ containing $0.15 \mathrm{M}$ sodium chloride (TBS). The sections were incubated at RT for 10 min with super block (SHP125) (ScyTek Laboratories, USA) to block nonspecific background staining. The sections were then covered with the primary antibodies diluted $1: 500$ for anti-caspase-3, $1: 200$ for anti-p53, 1 : 500 for anti-bcl-2 in TBS at $4^{\circ} \mathrm{C}$ overnight (Anti-caspase-3 (PA1302-1); anti-p53 (MA1078); antibcl-2 (MA1004) were from Boster Biological Technology Co. Ltd., USA). After washing in TBS for 15 min, the sections were incubated at RT for biotinylated link antibody (SHP125) (ScyTek Laboratories, USA). Then, treatment was followed with Streptavidin/HRP complex (SHP125) (ScyTek Laboratories, USA). Diaminobenzidine was used to visualize peroxidase activity in the tissues. Nuclei were lightly counterstained with haematoxylin, and then the sections were dehydrated and mounted. Both positive and negative controls were included in each run.

Light microscopy of immunohistochemically stained sections was performed by two pathologists, who were unaware of the patients' clinical information. Distribution, localization, and characteristics of immunostaining were recorded. Brown colour in the cytoplasm and/or nucleus of epithelial cells was evaluated as positive staining. Scoring was also performed by observers unaware of the patient data. Scoring differences between observers were resolved by consensus. For each antibody, the intensity of the reaction, negative $(-)$, weak $(1+)$, moderate $(2+)$ or strong $(3+)$, was determined in order to describe the immunoreactions.

\section{Statistical analysis}

SPSS version 16.0 for Windows (SPSS, Inc., Chicago, Illinois) was used for statistical analysis. To evaluate the distribution of variables, the Kolmogorov-Smirnov test was used. Parametric tests were used for normally distributed data and non-parametric tests were used for variables, which were not distributed normally. SD \pm values were used for parametric tests and \% values were used for non-paramet- 
ric tests as descriptive statistical values. The data were compared with the student $t$ test, and the Mann-Whitney $U$ test. A $p<0.05$ value was considered statistically significant.

\section{Results}

The mean age of patients was $66.0 \pm 10.9$ years in the BCC group, and $65.61 \pm 11.22$ years in the control group. Of the included patients, 18 (50.0\%) patients were female and 18 (50.0\%) patients were male. There was no significant difference among groups in terms of age or gender $(p>0.05)$. The baseline demographics and clinical characteristics of the study patients are summarized in Table 1.

The caspase-3 levels were statistically lower in the BCC group than in the control group $(p=0.033)$ (Figure 1 ). No significant differences were found between the BCC group and the control group in terms of $p 53$ levels ( $p=$ 1.000) or Bcl-2 levels ( $p=1.000$ ) (Table 2). P53, Bcl-2 and caspase-3 levels in the two groups are shown in Table 2.

\section{Discussion}

Eyelid tumours are the most commonly encountered neoplastic lesions in ophthalmology practice. Most eyelid tumours are benign in nature $[1,15]$; however, many malignant eyelid tumours also mimic benign lesions. This leads to a delay in diagnosis and possibly to severe morbidity or death. The most common malignant lesion of the eyelid is BCC, making up $90 \%$ of eyelid malignancies $[1,16]$. In the present study, all of the patients in the eyelid tumour group had BCC.

Table 1. Demographic data of all groups

\begin{tabular}{lccc}
\hline Parameter & Malignant & Control & $P$-value \\
\hline$N$ & 13 & 23 & \\
\hline Age, mean \pm SD [years] $^{*}$ & $66.00 \pm 10.89$ & $65.61 \pm 11.22$ & 1.000 \\
\hline${\text { Gender }(M / F)^{\&}}^{*}$ & $9 / 4$ & $9 / 14$ & 0.268 \\
\hline
\end{tabular}

*Student t test was used. \&Mann Whitney $U$ test was used.
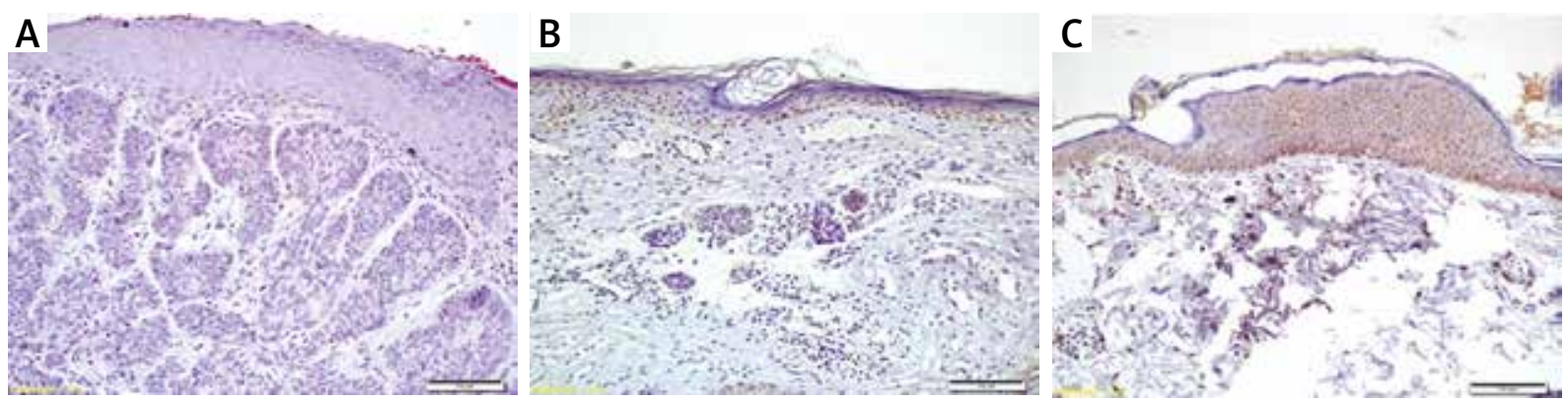

Figure 1. The expressions of caspase- 3 in the eyelid shown with immunochemical staining. A - Negative caspase-3 expression in basal cell carcinoma, $\mathbf{B}$ - grade 1 caspase-3 expression in basal cell carcinoma, $\mathbf{C}$ - grade 3 caspase-3 expression in controls

Table 2. Immunohistochemical staining of all groups

\begin{tabular}{|c|c|c|c|c|}
\hline Parameter & Immunohistochemical staining grade & Malignant & Control & $P$-value \\
\hline \multirow[t]{4}{*}{ p53 } & 0 & 4 & 3 & 1.000 \\
\hline & 1 & 5 & 13 & \\
\hline & 2 & 2 & 3 & \\
\hline & 3 & 2 & 1 & \\
\hline \multirow[t]{4}{*}{$\mathrm{Bcl}-2$} & 0 & 6 & 6 & 1.000 \\
\hline & 1 & 4 & 12 & \\
\hline & 2 & 2 & 5 & \\
\hline & 3 & 1 & 0 & \\
\hline \multirow[t]{4}{*}{ Caspase-3 } & 0 & 6 & 2 & 0.033 \\
\hline & 1 & 4 & 5 & \\
\hline & 2 & 1 & 11 & \\
\hline & 3 & 2 & 5 & \\
\hline
\end{tabular}

Mann Whitney $U$ test was used. 
Eyelid tumours affect elderly people more frequently [1]. This is thought to be due to the accumulative effects of cell injury and DNA damage, secondary to environmental factors. Ultraviolet radiation is the main environmental carcinogen and a common predisposing factor in eyelid tumours [17].

Apoptosis is a protective mechanism for cancer which leads damaged cells to death and prevents their expansion. Alterations in apoptotic and antiapoptotic proteins or imbalance between cell growth and apoptosis cause susceptibility to cancer [18].

The most intensively searched apoptotic protein in cancer is p53. It is the most researched molecule in carcinogenesis and it is lost or mutated in $50 \%$ of the human cancers [19]. In the present study, we stained p53 in malignant eyelid lesions and compared their staining levels with a control group but unlike other cancers, we found no significant difference in terms of p53 levels between these groups. These results may seem illogical, but this may be related to uneven numbers of BCC and control specimens. Also, in the present study, p53 staining was positive in $70 \%$ of the samples in the BCC group and it is similar with the literature data (42\% to 92\%) [20]. Demirkan et al. stained BCC samples and their adjacent normal epithelia for p53 and found out that there was no significant correlation between tumoral and normal epithelial cells in patients under the age of 65 [21]. Yalcin et al. found no significant difference in terms of $\mathrm{p} 53$ staining between BCC and actinic keratosis samples [22]. In another study, they found no correlation between $\mathrm{p} 53$ levels and BCC aggressiveness and recurrence [23]. But some studies show a significant correlation between p53 expression and BCC aggressiveness [24]. In the present study, we did not identify any BCC subtype and did not evaluate tumour aggressiveness and recurrence so we have no chance to compare our results with the most literature. But also most of the studies do not have any control group and therefore no comparison of p53 staining between malign and normal tissue.

$\mathrm{Bcl}-2$ was first researched in B-cell lymphoma and directly related to lymphoid carcinoma. We stained $\mathrm{BCl}-2$ in BCC samples and compared these with the control group. We found no significant difference in terms of staining intensity between the two groups. In the malignant group, $52 \%$ of samples were stained positive with Bcl- 2 and it was slightly lower than the literature data, in which Bcl-2 immunoreactivity was found to be between 67-100\% [25]. Sivrikoz et al. used immunohistochemical $\mathrm{Bcl}-2$ stain in $\mathrm{BCC}$ tissues and found out that decreased $\mathrm{BCl}-2$ levels were correlated with aggressive tumour subtypes [26]. We did not have any information about patients' BCC types and did not evaluate patients' clinical data for their prognosis. Also, our findings showed no difference between normal tissues and malignant lesions in terms of $\mathrm{Bcl}-2$ levels. So we cannot conclude about a relationship between $\mathrm{BCl}-2$ levels and eyelid cancer pathogenesis.
Caspase-3 is a key element of the apoptosis mechanism and most of the chemotherapeutics function through apoptosis. So, some of the researchers in the literature focus on the tumour resistance to chemotherapeutics and caspase- 3 relations. In a study, caspase-3 levels in BCC, SCC, and normal eyelid skin samples were measured by immunohistochemistry, western blot, and PCR techniques. They found lower staining intensity of caspase- 3 in the BCC samples than in the normal tissues [27]. In the present study, we evaluated caspase-3 staining intensity in two different groups and found out that caspase-3 staining was significantly lower in the malignant group than in the control group. As we can find, this was the second study about caspase-3 and skin malignancies and also the first to evaluate caspase- 3 levels in eyelid cancers. In the literature, there are some studies about caspase-3 correlation with gastrointestinal cancers and most of them stated that high caspase-3 levels lead to favourable prognosis [28]. In another study, they found significantly lower levels of caspase- 3 in prostatic carcinoma samples than the normal gland tissue. Also, Winter et al. stated that most poorly differentiated prostate cancers have reduced levels of caspase-3 [29]. Devarajan et al. studied breast cancer and pointed out that downregulation of caspase-3 may be related to chemoresistance of the cancer tissue [30]. In the present study, our finding is consistent with the literature, but we cannot conclude this is a certain result because of our small size of the BCC group. Nevertheless, these molecules may be important for basal cell carcinoma pathogenesis and need further and wider studies.

\section{Conclusions}

P53, Bcl-2 and caspase- 3 are regulatory proteins for apoptosis so they play an important role in cancer's pathophysiology and prognosis. Prior to the present study, there were no data in the literature about the levels of p53, Bcl-2 and caspase- 3 expressions in cancers of the eyelid. This study found caspase-3 levels to be lower in the BCC group than in the control group. This result may suggest that caspase-3 existed at lower levels and thus was unable to lead damaged cells to apoptosis and cause further mutations on these cells and eventually carcinogenesis. But the patient number was low in this study so we cannot obtain any certain results.

\section{Conflict of interest}

The authors declare no conflict of interest.

\section{References}

1. Deprez M, Uffer S. Clinicopathological features of eyelid skin tumors. A retrospective study of 5504 cases and review of literature. Am J Dermatopathol 2009; 31: 256-62.

2. Thompson CB. Apoptosis in the pathogenesis and treatment of disease. Science 1995; 267: 1456-62. 
3. Aubrey BJ, Kelly GL, Janic A, et al. How does p53 induce apoptosis and how does this relate to p53-mediated tumour suppression? Cell Death Differ 2018; 25: 104-13.

4. Momand J, Wu HH, Dasgupta G. MDM2: master regulator of the p53 tumor suppressor protein. Gene 2000; 242: 15-29.

5. Chen J. The cell-cycle arrest and apoptotic functions of p53 in tumor initiation and progression. Cold Spring Harb Perspect Med 2016; 6: a026104.

6. Erb P, Ji J, Wernli M, et al. Role of apoptosis in basal cell and squamous cell carcinoma formation. Immunol Lett 2005; 100: 68-72.

7. Czabotar PE, Lessene G, Strasser A, Adams JM. Control of apoptosis by the BCL-2 protein family: implications for physiology and therapy. Nat Rev Mol Cell Biol 2014; 15: 49-63.

8. Bakhshi A, Jensen JP, Goldman P, et al. Cloning the chromosomal breakpoint of $\mathrm{t}(14 ; 18)$ human lymphomas: clustering around $\mathrm{JH}$ on chromosome 14 and near a transcriptional unit on 18. Cell 1985; 41: 899-906.

9. Cohen GM. Caspases: the executioners of apoptosis. Biochem J 1997; 326: 1-16.

10. Boland K, Flanagan L, Prehn JH. Paracrine control of tissue regeneration and cell proliferation by caspase-3. Cell Death Dis 2013; 4: e725.

11. Xu M, Xia LP, Fan LJ, et al. Livin and caspase-3 expression are negatively correlated in cervical squamous cell cancer. Eur J Gynaecol Oncol 2013; 34: 152-5.

12. Huang KH, Fang WL, Li AF, et al. Caspase-3, a key apoptotic protein, as a prognostic marker in gastric cancer after curative surgery. Int J Surg 2018; 52: 258-63.

13. Chu J, Li JG, Joshi YB, et al. Gamma secretase-activating protein is a substrate for caspase-3: implications for Alzheimer's disease. Biol Psychiatry 2015; 77: 720-8.

14. D'Amelio M, Cavallucci V, Cecconi F. Neuronal caspase-3 signaling: not only cell death. Cell Death Differ 2010; 17: 1104-14.

15. Kersten RC, Ewing-Chow D, Kulwin DR, Gallon M. Accuracy of clinical diagnosis of cutaneous eyelid lesions. Ophthalmology 1997; 104: 479-84.

16. Bernardini FP. Management of malignant and benign eyelid lesions. Curr Opin Ophthalmol 2006; 17: 480-4.

17. Molho-Pessach V, Lotem M. Ultraviolet radiation and cutaneous carcinogenesis. Curr Probl Dermatol 2007; 35: 14-27.

18. Corręa Mde P, Ferreira AP, Gollner AM, et al. Markers expression of cell proliferation and apoptosis in basal cell carcinoma. An Bras Dermatol 2009; 84: 606-14.

19. Muller PA, Vousden KH, Norman JC. p53 and its mutants in tumor cell migration and invasion. J Cell Biol 2011; 192: 209-18.

20. Mercut R, Ciurea ME, margaritescu C, et al. Expression of p53, D2-40 and alpha-smooth muscle actin in different histological subtypes of facial basal cell carcinoma. Rom J Morphol Embryol 2014; 55: 263-72.

21. Demirkan NC, Colakoglu N, Düzcan E. Value of p53 protein in biological behavior of basal cell carcinoma and in normal epithelia adjacent to carcinomas. Pathol Oncol Res 2000; 6: 272-4.

22. Yalcin UK, Seckin S. The expression of p53 and COX-2 in basal cell carcinoma, squamous cell carcinoma and actinic keratosis cases. Turk Patoloji Derg 2012; 28: 119-27.

23. Healy E, Angus B, Lawrence CM, Rees JL. Prognostic value of Ki67 antigen expression in basal cell carcinomas. Br J Dermatol 1995; 133: 737-41.

24. Auepemkiate S, Boonyaphiphat P, Thongsuksai P. p53 expression related to the aggressive infiltrative histopathologi- cal feature of basal cell carcinoma. Histopathology 2002; 40: 568-73.

25. Ramdial PK, Madaree A, Reddy R, Chetty R. bcl-2 protein expression in aggressive and non-aggressive basal cell carcinomas. J Cutan Pathol 2000; 27: 283-91.

26. Sivrikoz NO, Kandiloğlu G. The effects of cyclin D1 and Bcl-2 expression on aggressive behavior in basal cell and basosquamous carcinoma. Iran J Pathol 2015; 10: 185-91.

27. Cousin F, Baldassini S, Bourchany D, et al. Expression of the pro-apoptotic caspase-3/CPP32 in cutaneous basal and squamous cell carcinomas. J Cutan Pathol 2000; 27: 235-41.

28. Chen $\mathrm{H}$, Yang X, Feng Z, et al. Prognostic value of caspase-3 expression in cancers of digestive tract: a meta-analysis and systematic review. Int J Clin Exp Med 2015; 8: 10225-34.

29. Winter RN, Kramer A, Borkowski A, Kyprianou N. Loss of caspase- 1 and caspase-3 protein expression in human prostate cancer. Cancer Res 2001; 61: 1227-32.

30. Devarajan E, Sahin AA, Chen JS, et al. Down-regulation of caspase-3 in breast cancer: a possible mechanism for chemoresistance. Oncogene 2002; 21: 8843-51. 